\title{
THE UNIFORM CLASSIFICATION OF BANACH SPACES
}

\author{
YOAV BENYAMINI \\ The Technion \\ Israel Institute of Technology \\ Haifa 32000 Israel \\ and \\ The University of Texas \\ Austin, Texas 78712
}

\begin{abstract}
This is a survey of results on the classification of Banach spaces as metric spaces. It is based on a series of lectures I gave at the Functional Analysis Seminar in 1984-1985, and it appeared in the 1984-1985 issue of the Longhorn Notes. I keep receiving requests for copies, because some of the material here does not appear elsewhere and because the Longhorn Notes are not so easy to get. Having it posted on the Bulletin thus seems reasonable despite the fact that it is not updated, and I thank the Editors of the Longhorn Notes for their permission to do so.
\end{abstract}

\section{$\S 0$. INTRODUCTION.}

Banach spaces are topological (metric) spaces with an additional structure the vector space structure. In the linear theory we study all these structures simultaneously, and we deal with linear continuous maps. Two spaces are identified if they are linearly homeomorphic. One could, however, consider Banach spaces as a special class of topological (or metric) spaces and study them as such. In the topological classification two spaces are identified if they are homeomorphic. In the metric classification we identify uniformly-homeomorphic spaces.

While the linear theory is very rich - there are many different types of spaces, with complicated subspace structure, the topological theory is, in some sense, trivial. A remarkable theorem of Kadec says that any two separable Banach spaces are homeomorphic. (See [BP] for a thorough study of Banach spaces as topological spaces.) Kadec's theorem was extended by Torunczyk $[\mathrm{T}]$, who proved that two Banach spaces are homeomorphic iff they have the same density character.

The uniform theory lies between these two extremes. It is rich enough so that to say that two Banach spaces are uniformly homeomorphic already says something about similarities in their linear structure. Yet it does not, in general, imply linear isomorphism.

In this series of lectures I presented some of the ideas and results in the theory of uniform classification. There is no attempt at a comprehensive survey. I chose one topic - the infinite dimensional classification problem, and presented, with

Partially supported by NSF Grant DMS 8403669.

Typeset by $\mathcal{A}_{\mathcal{M}} \mathcal{S}-\mathrm{T}_{\mathrm{E}} \mathrm{X}$ 
complete proofs, what I believe to be the main ideas and results on this subject. Even within this narrower subject I did not try to be exhaustive. For example, I didn't even mention when a result applies to more general spaces (linear metric spaces, Polish groups) - and many of the results do.

There are no new results here, but some of the proofs have been simplified a little (notably $\S 6$ ).

Let me mention briefly two topics on which I didn't speak and on which some significant work has been done.

The first is the problem of uniform embedding, which is very similar in spirit to the subject of these lectures. The following theorem of Aharoni [Ah] (see also [As] for a simpler proof) dramatically shows the difference between the linear and uniform theories. While $c_{0}$ is, linearly, a very "small" space, we have

Theorem 0.1. Every separable metric space Lipschitz embeds into $c_{0}$.

The second result, due to Aharoni, Maurey and Mityagin [AMM] gives a complete solution to the question which spaces embed uniformly into a Hilbert space.

Theorem 0.2. A linear metric space embeds uniformly into a Hilbert space iff it is linearly isomorphic to a subspace of $L^{0}$ - the space of measurable functions with the topology of convergence in measure.

In particular $L^{p}$ embeds uniformly into a Hilbert space iff $p \leq 2$.

For earlier results on the impossibility of uniform embedding of some spaces (including $c_{0}$ ) into a Hilbert space, see the very short and elegant papers of Enflo [E1, E2] as well as his [E3]. The first two papers already belong to the second subject I would like to mention - the local theory, or the theory of finite metric spaces. Rather than quote some of the results here, let me just say that there is a close similarity, in spirit and in methods, between this theory and the theory of finite dimensional Banach spaces, and refer the reader to few of the recent papers $[\mathrm{L}-\mathrm{J}],[\mathrm{Bo}],[\mathrm{B}-\mathrm{M}-\mathrm{W}]$ and their references.

We now briefly describe the content of these lectures.

The first four sections deal with Lipschitz classification and Lipschitz maps. This class of uniformly continuous functions is easier to deal with because under various circumstances they possess derivatives, and their derivatives are used to reduce the Lipschitz problem to a linear one.

We thus study, in the first two sections, the appropriate notions of differentiability and use these to obtain some strong results about the Lipschitz classification.

In the third section we prove Lindenstrauss' theorem on the linearization of Lipschitz retractions to yield linear projections (under suitable conditions). This is not always possible and we also show, following Lindenstrauss, that there is a Lipschitz retraction from $\ell_{\infty}$ onto $c_{0}$.

In $\S 4$ we present the main result of Heinrich and Mankiewicz [HM] which combines the techniques of $\S 1$ and $\S 3$ to show that "nice" separable spaces can be Lipschitz homeomorphic iff they are linearly isomorphic. This is not so for general spaces, however, and we also present the example of Aharoni-Lindenstrauss [AL1] of non-isomorphic Lipschitz homeomorphic Banach spaces.

In the last two sections we study general uniform homeomorphisms. Here the situation is much more delicate and difficult, because uniformly continous functions need not have derivatives. It turns out that uniformly homeomorphic spaces must have the same finite dimensional spaces. This result is due to Ribe [Ri1], [Ri2], and 
we present the simpler proof of Heinrich and Mankiewicz [HM]. We also give in this section a proof of an unpublished result of Enflo, that $\ell_{1}$ and $L_{1}$ are not uniformly homeomorphic.

The last section is devoted to a presentation of the recent examples of Ribe [Ri3] and Aharoni-Lindenstrauss [AL2] of uniformly homeomorphic and non-isomorphic uniformly convex, separable spaces.

\section{§1. Existance of DeRivatives.}

Let $f$ be a mapping from $X$ to $Y$, and $x_{0} \in X$. We say that $f$ is differentiable at $x_{0}$ if the limit $\frac{\partial f}{\partial x}\left(x_{0}\right)=\lim _{t \rightarrow \infty} \frac{1}{t}\left(f\left(x_{0}+t x\right)-f\left(x_{0}\right)\right)$ exists for each $x \in X$, and is linear in $x$. (This is usually called Gateaux differentiability, but we shall just call $f$ differentiable.) The linear map $x \rightarrow \frac{\partial f}{\partial x}\left(x_{0}\right)$ will be denoted by $(D f)_{x_{0}}(x)$.

Note that if $f$ is differentiable at $x_{0}$ and satisfies a Lipschitz condition with constant $K$, then $(D f)_{x_{0}}$ is a bounded linear operator with $\left\|(D f)_{x_{0}}\right\| \leq K$. Moreover, if $f$ also satisfies $\|f(x)-f(y)\| \geq \frac{1}{K}\|x-y\|$ for all $x, y \in X$ then $\left\|(D f)_{x_{0}}(x)\right\| \geq \frac{1}{K}\|x\|$, and $(D f)_{x_{0}}$ is an into isomorphism. We thus proved

Lemma 1.1. If $f: X \rightarrow Y$ is a Lipschitz embedding and $f$ is differentiable at $x_{0} \in X$, then $(D f)_{x_{0}}$ is a linear into isomorphism.

This simple Lemma motivates the study of differentiability of Lipschitz mappings, and the main result of this section is the following theorem, proved independently by N. Aronszajn [Ar], J.P.R. Christensen [C], and P. Mankiewicz [Man].

Theorem 1.2. A Lipschitz function from a separable Banach space $X$ to a Banach space $Y$ with the $R N P$ is differentiable at least at one point.

The requirement that $Y$ has the $R N P$ is essential for this type of result. Indeed, if $Y$ fails the $R N P$ there is a Lipschitz function $f: \mathbb{R} \rightarrow Y$ which is nowhere differentiable.

Many results on the impossibility of certain Lipschitz embeddings follow immediately from Lemma 1.1 and Theorem 1.2, and the known impossibility of linear embedding. The following examples were known before Theorem 1.2 was proved (Lindenstrauss [L] and Enflo [E1] and [E3]). But their original proofs were quite difficult and used special properties of the spaces involved.

(i) If $p<\infty$ and $q \neq p, \ell_{q}$ does not Lipschitz embed into $\ell_{p}$.

(ii) Unless $p=q=2$, or $p=\infty, L_{q}$ does not Lipschitz embed into $\ell_{p}$.

(iii) If $p<\infty, L_{q}$ does not Lipschitz embed into $L_{p}$ unless $2 \geq q \geq p \geq 1$ or $p=q$.

(The case $q>2, p=1$ does not follow from the Theorem because $L_{1}$ does not have the $R N P$. But it is still true that $L_{q}, q>2$ does not Lipschitz embed into $L_{1}$. See the Example at the end of $\S 2$.)

(iv) If $X$ Lipschitz embeds into a Hilbert space, it must be isomorphic to a Hilbert space. Indeed, Lemma 1.1 and Theorem 1.2 apply directly when $X$ is separable, and a space is isomorphic to a Hilbert space if all its separable subspaces are.

In order to prove Theorem 1.2 we first need to study the differentiability of Lipschitz functions from $\mathbb{R}$ or $\mathbb{R}^{n}$ into a space $Y$ with the $R N P$. This involves two classical results of Gelfand and of Rademacher. The first proposition was proved by I.N. Gelfand [G] when $Y$ is a separable dual. 
Proposition 1.3. A Lipschitz function $f$ from $\mathbb{R}$ into a space $Y$ with the $R N P$ is differentiable almost everywhere.

Proof. Consider the set function $\mu$ defined for intervals $[a, b]$ by $\mu([a, b])=f(b)-$ $f(a)$. As $f$ is a Lipschitz function, hence absolutely continuous and of bounded variation on each finite interval, $\mu$ can be extended in the standard way to a $\sigma$ additive Borel measure on $\mathbb{R}$ which is absolutely continuous with respect to the Lebesgue measure. As $Y$ satisfies the $R N P$, there is function $g: \mathbb{R} \rightarrow Y$, Bochner integrable on each finite interval so that $f(t)=f(0)+\int_{0}^{t} g(s) d s$.

Once this representation is given, the proof that $f$ is differentiable almost everywhere and that $f^{\prime}(t)=g(t)$ for almost all $t$ is the same as in the analogous situation for real valued functions.

The second proposition is a generalization of a famous theorem of H. Rademacher [Ra]. Rademacher proved it for $Y=\mathbb{R}^{m}$, but using Proposition 1.3 the generalization is only formal.

Proposition 1.4. A Lipschitz function $f$ from $\mathbb{R}^{n}$ into a space $Y$ with the $R N P$ is differentiable almost everywhere.

Proof. Recall that for a fixed $x \in \mathbb{R}^{n}$, we denote by $\frac{\partial f}{\partial x}(y)$ the derivative of $f$ at $y$ in the $x$ direction, i.e.,

$$
\frac{\partial f}{\partial x}(y)=\lim _{t \rightarrow 0}(f(y+t x)-f(y)) / t
$$

provided the limit exists.

By Proposition 1.3 this limit exists a.e. on each line parallel to $x$, thus by Fubini's Theorem it exists for almost all $y \in \mathbb{R}^{n}$.

Let $G$ be a countable dense additive subgroup of $\mathbb{R}^{n}$. By the above, the directional derivative $\frac{\partial f}{\partial g}(y)$ exists for almost all $y \in \mathbb{R}^{n}$ and for all $g \in G$, and we shall first show that except for a set of $y$ 's of measure zero it is linear in $g \in G$.

To this end let $\psi: \mathbb{R}^{n} \rightarrow \mathbb{R}$ be $C^{\infty}$ with compact support, and consider $f * \psi$. On the one hand $\frac{\partial}{\partial g}(f * \psi)=f * \frac{\partial \psi}{\partial g}$ which is linear in $g$ because $\psi$ is $C^{\infty}$.

On the other hand, by a simple change of variable

$$
\frac{\partial}{\partial g}(f * \psi)(y)=\lim _{t \rightarrow \infty} \int \frac{f(x+t g)-f(x)}{t} \psi(y-x) d x
$$

and as the functions $(f(x+t g)-f(x)) / t$ are bounded and converge a.e. to $\frac{\partial f}{\partial g}(x)$ this limit is also equal to $\frac{\partial f}{\partial g} * \psi$, hence, the latter is also linear in $g$, i.e., given any $g, h \in G,\left(\frac{\partial f}{\partial g}+\frac{\partial f}{\partial h}-\frac{\partial f}{\partial(g+h)}\right) * \psi \equiv 0$.

As $\psi$ is arbitrary and $G$ is countable it follows that for almost all $x \in \mathbb{R}^{n}$ we have

$$
\frac{\partial f}{\partial g}(x)+\frac{\partial f}{\partial h}(x)=\frac{\partial f}{\partial(g+h)}(x) \quad \text { for all } \quad g, h \in G
$$

Fix any $x$ for which $(*)$ holds. We shall show that in fact, it holds for all $g, h \in \mathbb{R}^{n}$ and not only in $G$, i.e., $f$ is differentiable at $x$. 
For $t>0$, put $f_{t}(y)=(f(x+t y)-f(x)) / t$. The family $\left(f_{t}\right)_{t>0}$ is equi-continuous, (in fact, they all satisfy the same Lipschitz condition that $f$ does). By the ArzellaAscolli Theorem this set is a relatively compact set of continuous functions. As it has a unique limit, when $t \rightarrow 0$, on the dense subset $G$ of $\mathbb{R}^{n}$, the limit exists for all $y \in \mathbb{R}^{n}$, and is continuous in $y$. As this continuous limit is linear on the dense subset $G$, it is linear everywhere. This completes the proof.

The proof of Theorem 1.2 will yield more than claimed. Not only do Lipschitz functions have derivatives at some points, but in fact, they are differentiable "almost everywhere." As an infinite dimensional space does not carry a "standard" measure one needs to define what is meant by "almost everywhere." Indeed, the main point in what N. Aronszajn, J.R.P. Christensen and P. Mankiewicz do is in the introduction of a useful notion of "almost everywhere," or equivalently of the complementary sets which we shall call "zero sets."

Different authors introduced different notions of zero sets, see $[\mathrm{A}],[\mathrm{M}],[\mathrm{C}],[\mathrm{P}]$, and we shall present the one introduced by J.P.R. Christensen [C], but they could all be used to prove Theorem 1.2, as they all satisfy the following definition.

Definition. Let $X$ be a Banach space. A family $\mathcal{N}$ of Borel subsets of $X$ is called a null family, and its members are called zero sets, if:

(i) $\mathcal{N}$ is closed under countable unions.

(ii) Let $A$ be a Borel set. If there exists a finite dimensional subspace $Y$ of $X$ so that $(A+x) \cap Y$ is a subset of $Y$ of Lebesgue measure zero for every $x$ in $X$, then $A$ is in $\mathcal{N}$.

(iii) Sets in $\mathcal{N}$ have empty interior.

(iv) If $Y$ is a finite dimensional subspace of $X$ then $A \in \mathcal{N}$ iff for all $x \in X,(A+x) \cap Y$ is a subset of $Y$ of Lebesgue measure zero.

Note that when $X$ is a finite dimensional space, the family of Borel subsets of $X$ of Lebesgue measure zero is a null family. In this case (iv) is a "mild" form of the Fubini Theorem. Notice that it is exactly this form of Fubini's Theorem that we used in deducing Proposition 1.4 from the one dimensional case, Proposition 1.3.

To emphasize the fact that only (i)-(iv) are needed to prove Theorem 1.2 and not the particular form of $\mathcal{N}$ we now complete the proof of the Theorem under the assumption that such a null family $\mathcal{N}$ exists on $X$ :

Proposition 1.5. Let $X$ be a separable Banach space and let $\mathcal{N}$ be a null family on $X$. Let $Y$ satisfy the RNP and assume $f: X \rightarrow Y$ is a Lipschitz function. Then $f$ is differentiable "almost everywhere", i.e., the set of points where $f$ is not differentiable belongs to $\mathcal{N}$.

Proof. Let $X_{1} \subset X_{2} \subset X_{3} \cdots$ be finite dimensional subspaces of $X$ so that $X=$ $\overline{\cup X_{n}}$. Define

$$
D_{n}=\left\{\begin{array}{l}
x \in X: \\
a \in X_{n}, \quad \text { and is linear in } a \in X_{n}
\end{array}\right\} .
$$

We claim that $X \backslash D_{n} \in \mathcal{N}$. By (iv) it suffices to show that for each fixed $z \in X,\left(\left(X \backslash D_{n}\right)+z\right) \cap X_{n}$ is a set of Lebesgue measure zero in $X_{n}$. But this set is exactly the set of points in $X_{n}$ where $g_{z}(x)=f(x+z)$ is non-differentiable as 
a function from $X_{n}$ to $Y$. Thus Proposition 1.4 implies that it has zero Lebesgue measure.

By (i) $\cup\left(X \backslash D_{n}\right)$ is also in $\mathcal{N}$, and we shall show that $f$ is differentiable at each $x \in \cap D_{n}$, i.e., in the complement of $\cup\left(X \backslash D_{n}\right)$. If $x \in \cap D_{n}$, $\frac{\partial f}{\partial a}(x)$ exists and is linear for $a$ 's in $\cup X_{n}$. As $\cup X_{n}$ is dense in $X$, the same argument used at the end of the proof of Proposition 1.4 shows that in fact $(D f)_{x}$ exists.

It remains to construct a null family $\mathcal{N}$, and we shall follow $[\mathrm{C}]$.

Definition. A Borel subset $A$ of $X$ is called a zero set, if there is a probability measure $\mu$ on $X$ (which we call a test measure for $A$ ), so that $\chi_{A} * \mu \equiv 0$, i.e., $\int \chi_{A}(x-y) d \mu(y)=0$ for all $x \in X$.

Note that this generalizes the notion of a zero set in $\mathbb{R}^{n}$. Indeed, denote the Lebesgue measure on $\mathbb{R}^{n}$ by $\lambda$. If $\chi_{A} * \mu \equiv 0$ for some Borel set $A$ and a probability measure $\mu$, integrating the equality with respect to $\lambda$ we obtain

$$
\begin{aligned}
0 & =\iint \chi_{A}(x-y) d \mu(y) d \lambda(x) \\
& =\int\left(\int \chi_{A}(x-y) d \lambda(x)\right) d \mu(y) \\
& =\int \lambda(A) d \mu(y)=\lambda(A) .
\end{aligned}
$$

Conversely, if $\lambda(A)=0$ take $\mu$ to be $\lambda$ restricted to the unit cube $C$. Then

$$
\begin{aligned}
\chi_{A} * \mu & =\int \chi_{A}(x-y) d \mu(y) \\
& =\int_{C} \chi_{A}(x-y) d \lambda(y) \\
& =\lambda((x-A) \cap C)=0 .
\end{aligned}
$$

We now have

Proposition 1.6. Let $X$ be a separable Banach space. Then the family of zero sets defined above is a null family.

Proof. To see the idea of the proof that $\mathcal{N}$ satisfies (i) let us first check that if $A, B \in \mathcal{N}$ so does $A \cup B$. Indeed, let $\mu, \nu$ be test measures for $A, B$ respectively, and let $\eta=\mu * \nu$. Then $\chi_{A} * \eta=\left(\chi_{A} * \mu\right) * \nu \equiv 0$ and similarly $\chi_{B} * \eta \equiv 0$. Hence $\chi_{A \cup B} * \eta \leq\left(\chi_{A}+\chi_{B}\right) * \eta \equiv 0$.

To carry this proof so that it applies to countable unions we need to form infinite convolutions, and this is where the completeness of $X$ is used. We first need some preparations.

Let $\mathcal{P}$ be the set of all Borel probability measures on $X$ with the $\omega^{*}$-topology induced by the bounded continuous functions on $X$. It is well known that $\mathcal{P}$ is separable and that its topology is given by a complete metric which is translation invariant, i.e., $d(\mu * \eta, \nu * \eta)=d(\mu, \nu)$ for all $\mu, \nu, \eta \in \mathcal{P}$. (See e.g., [Bi].)

Note that if $A \in \mathcal{N}$ and $\mu$ is a test measure for $A$, then so is every translate of $\mu$, and so is the restriction $\left.\frac{1}{\mu(B)} \mu\right|_{B}$ for any Borel subset $B$ so that $\mu(B)>0$. Thus by appropriate translation and restriction we can find a test measure for $A$ which 
is supported in an arbitrarily small ball centered at 0 , i.e., a test measure for which $d\left(\mu, \delta_{0}\right)$ is arbitrarily small.

Assume now that $A_{n} \in \mathcal{N}$ and let $\mu_{n}$ be test measures for $A_{n}$ with $d\left(\mu_{n}, \delta_{0}\right)<$ $2^{-n}$. Then $\mu_{1} * \ldots * \mu_{n}$ is a $d$-Cauchy sequence in $\mathcal{P}$ and let $\mu$ be its limit. For any $n, \mu=\mu_{n} * \eta_{n}$ where $\eta_{n}$ is the infinite convolution of all the $\mu_{k}$ 's except the $n^{\text {th }}$. Thus $\chi_{A_{n}} * \mu=\left(\chi_{A_{n}} * \mu_{n}\right) * \eta_{n} \equiv 0$, and hence also $\chi_{\cup A_{n}} * \mu \leq \sum \chi_{A_{n}} * \mu \equiv 0$, and $\cup A_{n} \in \mathcal{N}$. This proves (i), and (ii) is obvious. To prove (iii) assume $A \in \mathcal{N}$ has a non-empty interior. We can cover $X$ by a countable number of translates of $A$, and by (i) and (ii) this will yield that $X \in \mathcal{N}$. But this is impossible as $\chi_{X} * \mu \equiv \chi_{X}$ for all probability measures $\mu$.

It remains to check (iv). Let $\mu$ be a probability measure supported in $Y$ and equivalent to the Lebesgue measure on $Y$, and we shall check that it is a test measure for $A$. Indeed

$$
\begin{aligned}
\chi_{A} * \mu & =\int \chi_{A}(x-y) d \mu(y)=\mu(x-A) \\
& =\mu((x-A) \cap Y)=0 .
\end{aligned}
$$

This completes the proof of the Proposition, hence also of Theorem 1.2.

Remark. The methods of this section seem to yield only into linear isomorphisms even if the original Lipschitz map was an onto Lipschitz homeomorphism. If we knew that there is a point $x_{0}$ where $f$ is differentiable and so that, at the same time, $f^{-1}$ is differentiable at $f\left(x_{0}\right),(D f)_{x_{0}}$ would have been a linear onto isomorphism with inverse $\left(D f^{-1}\right)_{f^{-1}\left(x_{0}\right)}$. This led Christensen to raise the following problem: Assume $X$ and $Y$ are separable spaces and that $f: X \rightarrow Y$ is a Lipschitz homeomorphism. Does $f$ carry null sets in $X$ to null sets in $Y$ ? If the answer is positive, the results of this section imply that "most" points $x_{0}$ in $X$ will be such that both $f$ and $f^{-1}$ are differentiable in $x_{0}$ and $f\left(x_{0}\right)$ respectively.

\section{$\S 2 . \omega^{*}$-DERIVATIVES.}

It follows from Theorem 1.2 that Lipschitz mappings into separable dual spaces have derivatives, and since the derivative of a Lipschitz embedding is a linear isomorphism, it follows that when a separable space Lipschitz embeds into a separable dual $Y$, it embeds linearly into it.

In this section we show that this last result is true even when $Y$ is a nonseparable dual space. As $Y$ will no longer have to have the $R N P$, the Lipschitz embedding will not have to be differentiable anywhere. We shall define a weaker notion of a derivative - the $\omega^{*}$-derivative, and it will turn out that the embedding will have $\omega^{*}$-derivative "almost everywhere". The $\omega^{*}$-derivative will be a bounded linear operator, but it will not be automatically bounded from below. It turns out, however, that it will be bounded from below "almost everywhere". The results of this section are taken from S. Heinrich and P. Mankiewicz [HM].

Definition. Let $Y=Z^{*}$ be a dual space and $f: X \rightarrow Y$. We say that $f$ is $\omega^{*}$-differentiable at $x_{0} \in X$ if the $\omega^{*}$-limit

$$
\left(D^{*} f\right)_{x_{0}}(x)=\omega^{*}-\lim _{t \rightarrow 0}\left(f\left(x_{0}+t x\right)-f(x)\right) / t
$$


exists for all $x \in X$, and is linear in $x$. The linear mapping $\left(D^{*} f\right)_{x_{0}}$ is called the $\omega^{*}$-derivative of $f$ at $x_{0}$.

If $f$ satisfies a Lipschitz condition with constant $K$, the $\omega^{*}$-lower-semi-continuity of the norm implies that $\left(D^{*} f\right)_{x_{0}}$ is a linear operator of norm at most $K$.

Theorem 2.1. A Lipschitz map $f$ from a separable Banach space into the dual $Y=Z^{*}$ of a separable space $Z$, has a $\omega^{*}$-derivative almost everywhere. Moreover, if $f$ is a Lipschitz embedding, $\left(D^{*} f\right)_{x_{0}}$ is an isomorphism for almost all $x_{0}$.

Proof. By "almost everywhere" we mean, of course, "except for a zero set" in the sense described in the end of $\S 1$. As $Z$ is separable, most of the proof is routine extension of the ideas used in $\S 1$, applied to the scalar Lipschitz mappings $\varphi_{n}(x)=\left\langle z_{n}, f(x)\right\rangle$ where $\left(z_{n}\right)$ is a dense subset of the unit ball of $Z$. The only new ingredient is in the proof of the last part of the Theorem. Here also the whole idea can be seen in the one-dimensional case $X=\mathbb{R}$. The extension to general separable $X$ follows the same route taken in $\S 1$. Hence, we treat this case only. So assume $f: \mathbb{R} \rightarrow Z^{*}$ satisfies a Lipschitz condition with constant $K$, and without loss of generality normalize so that $\|f(t)-f(s)\| \geq|t-s|$ for all $t, s \in \mathbb{R}$. To simplify the notation write $f^{\prime}\left(t_{0}\right)$ for $\left(D^{*} f\right)_{t_{0}}$ when the latter exists. Thus, in this case

$$
\omega^{*}-\lim _{\lambda \rightarrow 0}\left(f\left(t_{0}+\lambda t\right)-f\left(t_{0}\right)\right) / \lambda=t f^{\prime}\left(t_{0}\right) .
$$

By the earlier parts of the Theorem we already know that $f^{\prime}(t)$ exists almost everywhere, and then, of course, $\left\|f^{\prime}(t)\right\| \leq K$. We need to show that $\left\|f^{\prime}(t)\right\| \geq 1$ for almost all $t$.

If this were false, find $\delta<1$ so that $A=\left\{t:\left\|f^{\prime}(t)\right\| \leq \delta\right\}$ has positive measure, and let $t_{0}$ be a density point of $A$. Fix $\epsilon>0$ so small that

$$
m\left(\left[t_{0}, t_{0}+\epsilon\right] \cap A\right) \geq \epsilon\left(1-\frac{1-\delta}{3 K}\right)
$$

and let $B=\left[t_{0}, t_{0}+\epsilon\right] \backslash A$. Then $m(B) \leq \epsilon \frac{1-\delta}{3 K}$ and $\left\|f^{\prime}(t)\right\| \leq K$ on $B$.

As $\left\|f\left(t_{0}+\epsilon\right)-f\left(t_{0}\right)\right\| \geq \epsilon$, find $z \in Z,\|z\|=1$ with $\left\langle z, f\left(t_{0}+\epsilon\right)-f\left(t_{0}\right)\right\rangle \geq$ $\epsilon(1+\delta) / 2$, and define $\varphi(t)=\langle z, f(t)\rangle . \varphi$ is a scalar Lipschitz function, hence the integral of its derivative. Thus

$$
\begin{aligned}
\epsilon(1+\delta) / 2 & \leq \varphi\left(t_{0}+\epsilon\right)-\varphi\left(t_{0}\right)=\int_{t_{0}}^{t_{0}+\epsilon} \varphi^{\prime}(t) d t \\
& \leq \int_{B}\left\|\varphi^{\prime}(t)\right\|+\int_{A}\left\|\varphi^{\prime}(t)\right\| \\
& \leq K m(B)+\epsilon \delta \leq \epsilon\left(\frac{1-\delta}{3}+\delta\right)
\end{aligned}
$$

a contradiction.

Theorem 2.1 applies to dual of separable spaces. But for our purposes the separability requirement can be overcome by the following lemma which is of interest for its own sake. 
Lemma 2.2. Let $E$ be a separable subspace of the dual space $F^{*}$. Then there is a subspace $Y$ of $F^{*}$, containing $E$ which is isomorphic to the dual of a separable space $Z$.

For the proof, see [HM], Proposition 3.4.

Combining this result with Theorem 2.1 we obtain

Corollary 2.3. Let $X$ be a separable Banach space. If $X$ Lipschitz embeds into a dual space, it embeds linearly into it. In particular, if $X$ Lipschitz embeds into any space $Y$, it embeds linearly into $Y^{* *}$.

Proof. Assume $f: X \rightarrow F^{*}$ is a Lipschitz embedding. As $X$ is separable $f(X)$ is contained in a separable subspace $E$ of $X^{*}$, and find $Y, Z$ as in Lemma 2.2. Now use Theorem 2.1 to find $x_{0}$, so that $\left(D^{*} f\right)_{x_{0}}$ is a linear isomorphism into $Y$. (Note that $\left(D^{*} f\right)_{x_{0}}$ is taken with respect to the $\omega^{*}$ topology on $Y=Z^{*}$ and not with respect to the original $\omega^{*}$ topology on $F^{*}$.)

Example. We can now show that $L_{q}$ does not Lipschitz embed in $L_{1}$ for $q>2$. Indeed, by the Corollary this would have implied that $L_{q}$ embeds linearly into the $L$-space $L_{1}^{* *}$ which is impossible.

\section{§3. Linearization of Lipschitz RETRACTIONS.}

The main result of this section is the following Theorem of J. Lindenstrauss [L].

Theorem 3.1. Let $X$ be a subspace of $Y$ so that there is a Lipschitz retraction from $Y$ onto $X$. If $X$ is complemented in $X^{* *}$ it is complemented in $Y$.

Before we give the detailed proof let us explain the idea. Assume $f: Y \stackrel{\text { onto }}{\longrightarrow} X$ is the Lipschitz retraction, and that $f$ is differentiable at a point $x_{0} \underline{\operatorname{in} X}$. Then $(D f)_{x_{0}}$ is a linear operator from $Y$ into $X$ which is a projection. Indeed, $f$ is the identity on $X$, thus for every $x \in X$ and every $t,\left(f\left(x_{0}+t x\right)-f\left(x_{0}\right)\right) / t=x$, hence also $(D f)_{x_{0}}(x)=x$.

Our assumptions do not guarantee that $f$ is differentiable anywhere - and certainly not in a point of the "small" subset $X$ of $Y$. We thus need a "smoothing" procedure. This will be done on each finite dimensional subspace of $Y$ (see Lemma 3.3 ), and then we shall use a routine compactness argument to obtain an operator $T: Y \rightarrow X^{* *}$ so that $T$ is the identity on $X$. As $X$ is complemented in $X^{* *}$, say by a projection $P, P T: Y \rightarrow X$ will be the desired projection.

¿From this description it might appear that the assumption that $X$ is complemented in $X^{* *}$ is inessential, and is just a technical drawback of our method of proof. But this is not the case as the following example of Lindenstrauss [L] shows.

Example. There is a Lipschitz retraction from $\ell_{\infty}$ onto $c_{0}$.

Given $x=\left(a_{1}, \ldots, a_{n}, \ldots\right) \in \ell_{\infty}$, denote by $d(x)$ its distance to $c_{0}$, i.e., $d(x)=$ $\lim \sup \left|a_{n}\right|$. Define now $f: \ell_{\infty} \rightarrow c_{0}$ by

$$
(f(x))(n)=\left\{\begin{array}{ll}
0 & \left|a_{n}\right| \leq d(x) \\
\frac{a_{n}}{\left|a_{n}\right|}\left(\left|a_{n}\right|-d(x)\right) & \left|a_{n}\right|>d(x)
\end{array} .\right.
$$

One easily checks that $f$ is a retraction from $\ell_{\infty}$ onto $c_{0}$ and that it satisfies a Lipschitz condition with constant 2.

The example above is in fact a special case of a more general theorem of Lindenstrauss [L] which we just quote without proof. 
Theorem 3.2. If $K$ is a compact metric space then $C(K)$ is an absolute Lipschitz retract, i.e., if $Y$ is any metric space containing $C(K)$ there is a Lipschitz retraction from $Y$ onto $C(K)$.

We now pass to the proof of Theorem 3.1. The main step in the proof is contained in the following Lemma, where Lipschitz maps on finite dimensional spaces are "linearized".

Lemma 3.3. Let $Z$ be a finite dimensional space and let $E$ be a subspace of $Z$. Let $f$ be a Lipschitz function from $Z$ into a Banach space $X$ so that $\left.f\right|_{E}$ is a given linear operator $S$. Then there is a linear operator $T: Z \rightarrow X^{* *}$ so that $\left.T\right|_{E}=S$ and whose norm is at most the Lipschitz constant of $f$.

Proof. Write (algebraically) $Z=E \oplus Y$, and assume $\operatorname{dim} Y=m$ say. We first claim that without loss of generality we can assume that the directional derivatives $\frac{\partial f}{\partial e}$ in the direction of $e \in E$ exist everywhere, are continuous in $z$, and are linear in $e \in E$.

Indeed, let $\varphi \geq 0$ be a $C^{\infty}$ function on $E$ with compact support so that $\int \varphi=1$ and $\varphi(x)=\varphi(-x)$ for all $x \in E$, and replace $f$ by the function $F$ given by

$$
F(z)=\int_{E} f(z+x) \varphi(x) d x .
$$

It is easy to check (using change of variable as below) that $F$ is differentiable as required. To check the continuity of the directional derivatives, fix $e \in E$ and $t$. By a change of variable write

$$
F(z+t e)=\int_{E} f(z+x) \varphi(x-t e) d x .
$$

Thus for $z, z^{\prime} \in Z$ we have

$$
\begin{aligned}
\|(F(z+t e)-F(z)) / t-(F & \left.\left(z^{\prime}+t e\right)-F\left(z^{\prime}\right)\right) / t \|= \\
& =\left\|\int_{E}\left(f(z+x)-f\left(z^{\prime}+x\right)\right)(\varphi(x-t e)-\varphi(x)) / t d x\right\| \\
& \leq K\left\|z-z^{\prime}\right\| \int_{E}\|(\varphi(x-t e)-\varphi(x)) / t\| d x
\end{aligned}
$$

(where $K$ is the Lipschitz constant of $f$ ). Passing to the limit as $t \rightarrow 0$ gives

$$
\left\|\frac{\partial F}{\partial e}(z)-\frac{\partial F}{\partial e}\left(z^{\prime}\right)\right\| \leq K\left\|z-z^{\prime}\right\| \int_{E}\left\|\frac{\partial \varphi}{\partial e}(x)\right\| d x
$$

hence $\frac{\partial F}{\partial e}$ satisfies a Lipschitz condition.

To see that the restriction of $F$ to $E$ is $S$, we use the linearity of $\left.f\right|_{E}$ : If $e \in E$,

$$
\begin{aligned}
F(e) & =\int_{E} f(e+x) \varphi(x) d x \\
& =\int_{E}(f(e)+f(x)) \varphi(x) d x=f(e)
\end{aligned}
$$


because $\int \varphi(x)=1$ and $\int f(x) \varphi(x) d x=0$ because $f$ is linear on $E$, so $f(x)=$ $-f(-x)$ while $\varphi(x)=\varphi(-x)$.

¿From now on we can thus assume $f$ is already "smooth" in the $E$ directions.

To prove the Lemma we shall now use a "smoothing kernel" in the $Y$ direction. Let $\psi \geq 0$ be a $C^{\infty}$ function on $Y$ with compact support so that $\int_{Y} \psi=1$. Define

$$
f_{n}(z)=n^{m} \int_{Y} f(z+y) \psi(n y) d y .
$$

As $f$ is already smooth in the $E$ directions, each $f_{n}$ is differentiable so let $T_{n}=$ $\left(D f_{n}\right)_{0}$ be its differential at 0 . The sequence of operators $\left(T_{n}\right)$ is uniformly bounded from $Z$ to $X \subset X^{* *}$ so fixing a free ultra-filter $U$ on $\mathbf{N}$ define $T z=\omega^{*}-\lim _{U} T_{n} z$. We need only check that $\left.T\right|_{E}=S$. So fix $e \in E$, and then $T e=\lim T_{n} e=$ $\lim \frac{\partial f_{n}}{\partial e}(0)$. But

$$
\frac{\partial f_{n}}{\partial e}(0)=n^{m} \int_{Y} \frac{\partial f}{\partial e}(y) \psi(n y) d y=\int_{Y} \frac{\partial f}{\partial e}\left(n^{-1} y\right) \psi(y) d y
$$

and, of course, $\frac{\partial f}{\partial e}(0)=S e$ because $\left.f\right|_{E}=S$. Thus

$$
T e-S e=\lim \int_{Y}\left(\frac{\partial f}{\partial e}\left(n^{-1} y\right)-\frac{\partial f}{\partial e}(o)\right) \psi(y) d y
$$

which is zero because $\psi$ has a compact support and $\frac{\partial f}{\partial e}$ is continuous.

Proof of Theorem 3.1. The proof now follows easily from the Lemma. Let $Y_{\alpha}$ be a net, directed by inclusion, of finite dimensional subspaces of $Y$ so that $Y=\cup Y_{\alpha}$. For each $\alpha$ use the Lemma with $Z=Y_{\alpha}$ and $E=E_{\alpha}=Y_{\alpha} \cap X \subset Z$. Note that since $f$ is a retraction of $Y$ onto $X,\left.f\right|_{E_{\alpha}}$ is the identity on $E_{\alpha}$. By the Lemma, find a linear operator $T_{\alpha}: Y_{\alpha} \rightarrow X^{* *}$ so that $\left.T_{\alpha}\right|_{X \cap Y_{\alpha}}$ is the identity on this space, and so that $\left\|T_{\alpha}\right\| \leq K$, the Lipschitz constant of $f$. By compactness $\left(T_{\alpha}\right)$ has a $\omega^{*}$ convergent subnet, thus its limit $T$ is an operator from $Y$ to $X^{* *}$ with $\left.T\right|_{X}=i d_{X}$. Now take $P T: Y \rightarrow X$ as the desired projection, where $P: X^{* *} \rightarrow X$ is the given bounded linear projection.

\section{§4. LineAR ISOMORPHISM BETWEEN LIPSCHITZ EQUiVALENT SPACES.}

The differentiation theory of $\S 1$ yields that if two "nice" spaces are Lipschitz equivalent, they embed linearly into each other, but we couldn't prove that they are actually isomorphic (see the Remark at the end of $\S 1$ ). It turns out, however, that one can combine the results of $\S 1$ with the retraction linearization of $\S 3$ to obtain that the "nice" spaces embed linearly as complemented subspaces of each other. Thus, we obtain linear isomorphism results for a large class of spaces for which the "decomposition scheme" holds. After presenting this method, which is due to S. Heinrich and P. Mankiewicz [HM], we present the example of I. Aharoni and J. Lindenstrauss [AL1] of two ("non-nice") spaces which are Lipschitz equivalent but not linearly isomorphic. 
Theorem 4.1. Let $f$ be a Lipschitz embedding of $X$ into a space $Y$ so that there is a Lipschitz retraction from $Y$ onto the image $f(X)$. Assume that $f$ is differentiable at a point $x_{0} \in X$ and that $X$ is a Lipschitz retract of $X^{* *}$. Then there is a Lipschitz retraction from $Y$ onto $(D f)_{x_{0}}(X)$. In particular, if $X$ is linearly complemented in $X^{* *}$, there is a linear projection from $Y$ onto $(D f)_{x_{0}}(X)$.

Proof. The last claim follows from the first and Theorem 3.1, so we need only prove the first. WLOG $x_{0}=0$ and $f(0)=0$, and denote $(D f)_{0}$ by $D$. Let $\pi: Y \rightarrow f(X)$ be the Lipschitz retraction and define $g$ and $g_{n}$ from $Y$ into $X$ by $g=f^{-1} \circ \pi$ and $g_{n}(y)=n g(y / n)$. The functions $g_{n}: Y \rightarrow X \subset X^{* *}$ are uniformly Lipschitz and for each $y \in Y$ the sequence $g_{n}(y)$ is bounded. We can thus find a $\omega^{*}$-limit point $h: Y \rightarrow X^{* *}$ of the $g_{n}$ 's which, by the $\omega^{*}$-lower semi-continuity of the norm is again Lipschitz with the same constant.

We claim that $h(D x)=x$ for all $x \in X$. Indeed, put $y=D x$, i.e., $y=$ $\lim _{n} n f(x / n)$. As the $g_{n}$ 's are uniformly Lipschitz, we deduce that

$$
g_{n}(y)-g_{n}(n f(x / n)) \rightarrow 0 .
$$

But $g_{n}(y)$ has a subnet converging $\omega^{*}$ to $h(y)$ while

$$
g_{n}(n f(x / n))=n g(f(x / n))=x,
$$

i.e., $x=h(y)=h(D x)$. The desired retraction is now $D \circ \rho \circ h$, where $\rho$ is the retraction from $X^{* *}$ onto $X$.

Next we formulate one isomorphism result that follows from the Theorem. We refer the reader to $[\mathrm{HM}]$ for many more variations and refinements of the same theme.

Corollary 4.2. Let $X$ and $Y$ be separable reflexive spaces isomorphic to their squares. If $X$ and $Y$ are Lipschitz equivalent, they are linearly isomorphic.

Proof. By Theorem 1.2 all the conditions of Theorem 4.1 are satisfied. Thus $X$ and $Y$ are each linearly isomorphic to a complemented subspace of the other, and the result follows from Pełczyǹski's decomposition method.

As the next example shows, some conditions are necessary to deduce linear isomorphism from Lipschitz equivalence. But the spaces in the example are not "nice". They are non-separable and do not have the Radon-Nykodim property. In particular it is unknown if separable examples exist or if reflexive examples exist.

Example. ([AL1]). There are Lipschitz equivalent spaces $X$ and $Y$ which are not linearly isomorphic. In fact $Y$ does not embed linearly into $X$.

Let $\left\{N_{\gamma}: \gamma \in \Gamma\right\}$ be an uncountable collection of infinite subsets of natural numbers so that $N_{\gamma} \cap N_{\beta}$ is finite for all $\gamma \neq \beta$. Take $X$ to be the subspace of $\ell_{\infty}$ spanned by $c_{0}$ and the characteristic functions $\chi_{N_{\gamma}}$ of the sets $N_{\gamma}$. Let $Y$ be $c_{0}(\Gamma)$. The coordinate functionals on $\ell_{\infty}$ are a countable family in $X^{*}$ which separates the points of $X$. As no countable family in $Y^{*}$ separates the points of $Y$, it cannot be linearly embedded into $X$.

The quotient space $X / c_{0}$ is isometric to $c_{0}(\Gamma)$. Indeed, for any $\gamma_{1}, \ldots, \gamma_{n} \in \Gamma$ and $a_{1}, \ldots, a_{n}$,

$$
\left\|\sum_{1}^{n} a_{j} \chi_{N_{\gamma_{j}}}\right\|_{X / c_{0}}=\max \left|a_{j}\right|
$$


i.e., the images of $\chi_{N_{\gamma}}$ in the quotient are isometrically equivalent to the unit vectors of $c_{0}(\Gamma)$. Let $q: X \rightarrow X / c_{0}$ be the quotient map. The heart of the construction is to show that $q$ admits a Lipschitz "lifting", i.e., there is a Lipschitz map $f: X / c_{0} \rightarrow X$ so that $q \circ f$ is the identity on $X / c_{0}$. Once this is shown it follows easily that $X$ and $Y$ are Lipschitz equivalent. Indeed, $X$ is Lipschitz equivalent to $c_{0} \oplus X / c_{0}$ via the map $x \rightarrow(x-f(q(x)), q(x))$ whose inverse is $(y, z) \rightarrow y+f(z)$. But $c_{0} \oplus X / c_{0}=c_{0} \oplus c_{0}(\Gamma)$ is isometric to $c_{0}(\Gamma)=Y$.

We shall define $f$ on $c_{0}(\Gamma)^{+}$, the nonnegative elements of $c_{0}(\Gamma)$ so as to satisfy $\|f(y)-f(t)\| \leq\|y-z\|$ whenever $y, z \in c_{0}(\Gamma)^{+}$. We shall then define, for any $y \in c_{0}(\Gamma), f(y)=f\left(y^{+}\right)-f\left(y^{-}\right)$where $y=y^{+}-y^{-}$is the cannonical representation of $y$ as a difference of two disjointly supported nonnegative terms. It follows that

$$
\begin{aligned}
\|f(y)-f(z)\| & \leq\left\|f\left(y^{+}\right)-f\left(z^{+}\right)\right\|+\left\|f\left(z^{-}\right)-f\left(y^{-}\right)\right\| \\
& \leq 2 \max \left(\left\|y^{+}-z^{+}\right\|,\left\|y^{-}-z^{-}\right\|\right) \leq 2\|y-z\|
\end{aligned}
$$

and $f$ is a Lipschitz map with constant 2. Thus fix $y \geq 0$ in $c_{0}(\Gamma)$ and assume $y=\sum_{j=1}^{\infty} a_{j} e_{\gamma_{j}}$ where $a_{1} \geq a_{2} \geq \cdots$.

Let $M_{1}=N_{\gamma_{1}}$ and define inductively $M_{n}=N_{\gamma_{n}} \backslash \cup_{j<n} N_{\gamma_{j}}$. Define now

$$
f(y)=\sum a_{j} \chi_{M_{j}}
$$

Note that each $\chi_{M_{j}} \in X$ as it differs from $\chi_{N_{\gamma_{j}}}$ by a $c_{0}$ element. Also $q\left(\chi_{M_{j}}\right)=$ $q\left(\chi_{N_{\gamma_{j}}}\right)=e_{\gamma_{j}} \in c_{0}(\Gamma)$, so $q(f(y))=y$. To see that $f$ satisfies a Lipschitz condition we give another formula for $f(y)$ : Given $n,(f(y))_{n}$, the $n^{t h}$ coordinate of $f(y)$, is equal to $a_{i}$ iff $n \in N_{\gamma_{i}}$ but $n \notin N_{\gamma_{1}} \cup \cdots \cup N_{\gamma_{i-1}}$. So let $A_{n}$ be the closed subspace of $c_{0}(\Gamma)$ given by $A_{n}=\overline{s p}\left\{e_{\gamma}: n \in N_{\gamma}\right\}$. It follows from the above and the monotonicity of the $a_{i}$ 's that if $y \in c_{0}(\Gamma)^{+}$we have:

$$
(f(y))_{n}=\operatorname{dist}\left\{y, A_{n}\right\}
$$

It is clear from this formula that given $y, z \in c_{0}(\Gamma)^{+}$,

$$
\begin{aligned}
& \qquad\left|(f(y))_{n}-(f(z))_{n}\right|=\left|\operatorname{dist}\left\{y, A_{n}\right\}-\operatorname{dist}\left\{z, A_{n}\right\}\right| \leq\|y-z\|, \\
& \text { i.e., }\|f(y)-f(z)\| \leq\|y-z\| \text {. }
\end{aligned}
$$

\section{$\S 5$. UNIFORM HOMEOMORPHISMS.}

As uniformly continuous functions do not have derivatives in general, their study requires bona fide metric, geometric and topological arguments, and usually cannot be reduced to the linear theory.

The following simple lemma is usually the tool by which we get some initial control on a uniformly continuous function. We say that $f: X \rightarrow Y$ is Lipschitz for large distances if for each $\delta>0$ there is a $K=K(\delta)$ so that $\|f(x)-f(y)\| \leq$ $K\|x-y\|$ for all $x, y \in X$ satisfying $\|x-y\| \geq \delta$. 
Lemma 5.1. A uniformly continuous function between Banach spaces is Lipschitz for large distances.

Proof. Given $\delta$, choose first $M$ so that $\|f(a)-f(b)\| \leq M$ for all $a, b \in X$ satisfying $\|a-b\|<\delta$, and let $K(\delta)=2 M / \delta$. Given $x, y$ with $\|x-y\| \geq \delta$, let $x=a_{0}, a_{1}, \ldots, a_{m}=y$ be points in $X$ so that $\left\|a_{j+1}-a_{j}\right\|<\delta$, and $m=[2\|x-y\| / \delta]$. Then

$$
\|f(x)-f(y)\| \leq \sum_{i=1}^{m}\left\|f\left(a_{i}\right)-f\left(a_{i-1}\right)\right\| \leq m M \leq K(\delta)\|x-y\| .
$$

The next theorem (from $[\mathrm{HM}]$ ) will enable us to use some of the tools that we developed for Lipschitz maps in the uniformly continuous case.

Theorem 5.2. If $X$ and $Y$ are uniformly homeomorphic, they have Lipschitz equivalent ultra powers.

Proof. Let $f: X \rightarrow Y$ be a uniform homeomorphism. By Lemma 5.1 there is a constant $K$ so that $\left\|f\left(x_{1}\right)-f\left(x_{2}\right)\right\| \leq K\left\|x_{1}-x_{2}\right\|$ and

$$
\left\|f^{-1}\left(y_{1}\right)-f^{-1}\left(y_{2}\right)\right\| \leq K\left\|y_{1}-y_{2}\right\|
$$

for all $x_{1}, x_{2} \in X$ and $y_{1}, y_{2} \in Y$ satisfying $\left\|x_{1}-x_{2}\right\|,\left\|y_{1}-y_{2}\right\| \geq 1$. Define $f_{n}(x)=\frac{1}{n} f(n x)$. Then the modulus of continuity of $f_{n}$ is not worse than that of $f$, and $f_{n}$ already satisfies the $K$-Lipschitz condition $\left\|f_{n}\left(x_{1}\right)-f_{n}\left(x_{2}\right)\right\| \leq K\left\|x_{1}-x_{2}\right\|$ whenever $\left\|x_{1}-x_{2}\right\| \geq \frac{1}{n}$. As $f_{n}^{-1}(y)=\frac{1}{n} f^{-1}(n y)$ the same remarks apply to $f_{n}^{-1}$.

Let $U$ be a free ultra filter on the natural numbers, and define $F=\left(f_{n}\right)$ to be the natural map from $(X)_{U}$, the ultra power of $X$ onto $(Y)_{U}$.

We shall show that $F$ satisfies a Lipschitz condition with constant $K$. Fix $x=$ $\left(x_{1}, \ldots\right)$ and $z=\left(z_{1}, \ldots\right)$ in $(X)_{u}$, and choose, by uniform continuity of $f$, a $\delta>0$ so that $\|f(a)-f(b)\| \leq K\|x-z\|$ for all $a, b \in X$ satisfying $\|a-b\|<\delta$. Then we also have $\left\|f_{n}(a)-f_{n}(b)\right\| \leq K\|x-z\|$ for all $n$. As there are only finitely many $n$ 's for which $\delta \leq\left\|x_{n}-z_{n}\right\| \leq \frac{1}{n}$, we see, by the choice of $\delta$ and $K$, that for each $\epsilon>0, \quad\left\|f_{n}\left(x_{n}\right)-f_{n}\left(z_{n}\right)\right\| \leq K\|x-z\|+\epsilon$ for all but finitely many $n$ 's, i.e., $\|F(x)-F(z)\| \leq K\|x-z\|$. Similar arguments hold for $F^{-1}$.

We shall now deduce two results of Ribe [Ri1], [Ri2] from Theorem 5.2. These are only two examples of the consequences of Theorem 5.2 and its variations, and we refer the reader to the fundamental paper of Heinrich and Mankiewicz [HM] for many more details.

Theorem 5.3. If $X$ is uniformly homeomorphic to a $\mathcal{L}_{p}$ space, $1<p<\infty$, then it is a $\mathcal{L}_{p}$ space itself.

Proof. $X$ is a $\mathcal{L}_{p}$ space iff $(X)_{u}$ is such a space for some ultra filter $U$, and by Theorem 5.2 there is a $U$ for which $(X)_{u}$ is Lipschitz equivalent to an ultra power of a $\mathcal{L}_{p}$ space, i.e., to a $\mathcal{L}_{p}$ space. It thus remains to show that a space $Z$, Lipschitz equivalent to a $\mathcal{L}_{p}$ space $Y$ is itself a $\mathcal{L}_{p}$ space. Let $f: Z \rightarrow Y$ be the uniform homeomorphism.

If $Z$ is separable, this follows immediately from Theorems 1.2 and 4.1. Indeed, $Y$ is reflexive (because $1<p<\infty$ ), so $f$ has a point of differentiability, hence $Z$ is isomorphic to a complemented subspace of $Y$. 
The general case is reduced easily to the separable one, by showing that every separable subspace $Z_{0} \subset Z$ is contained in a separable subspace $W \subset Z$ which is Lipschitz equivalent to a $\mathcal{L}_{p}$ space. Indeed, define inductively sequences $Z_{0} \subset$ $Z_{1} \subset \cdots$ in $Z$ and $Y_{0} \subset Y_{1} \subset \cdots$ in $Y$ as follows: Having defined $Z_{n}$, let $Y_{n}$ be a separable $\mathcal{L}_{p}$ subspace of $Y$ containing $f\left(Z_{n}\right)$, and having defined $Y_{n}$ let $Z_{n+1}$ be any subspace of $Z$ containing $f^{-1}\left(Y_{n}\right)$. Then $\overline{\cup Y_{n}}$ is a $\mathcal{L}_{p}$ space, Lipschitz equivalent to $W=\overline{\cup Z_{n}}$.

Theorem 5.4. If $X$ and $Y$ are uniformly homeomorphic, there is a constant $C \geq 1$ so that for each finite dimensional subspace $E$ of $X$ there is a subspace $F$ of $Y$ with $d(E, F) \leq C$.

Proof. By Theorem 5.2 there is an ultra filter $U$ and a Lipschitz equivalence $f:(X)_{u} \rightarrow(Y)_{u}$. Fixing $E \subset X$ and considering it as a subspace of $(X)_{u}$, we have by Corollary 2.3 that $E$ embeds linearly into $\left((Y)_{u}\right)^{* *}$. The result now follows from local reflexivity and the local structure of ultra powers.

Theorem 5.4 gives many examples of non-uniformly homeomorphic Banach spaces and leads naturally to the question of whether some converse to it holds, i.e., whether identical local structures for two separable spaces imply that they are uniformly homeomorphic. As the following example shows there must be some additional requirements for such a converse to hold. The example is due to P. Enflo (unpublished) and was shown to me by J. Lindenstrauss.

Example. $L_{1}$ and $\ell_{1}$ are not uniformly homeomorphic.

We start with some observations for general Banach spaces, and then specialize to $L_{1}$ and $\ell_{1}$.

Assume $f: X \rightarrow Y$ is a uniform homeomorphism. As $f^{-1}$ is uniformly continuous it is Lipschitz for large distances, and there is a constant $L>0$ so that

$$
\|x-y\| \leq \max \{1, L\|f(x)-f(y)\|\}
$$

for all $x, y \in X$.

Also, for each $\delta>0$, let $K(\delta)$ be the smallest Lipschitz constant of $f$ for distances above $\delta$, i.e., the smallest constant $K(\delta)$ so that

$$
\|x-y\| \leq \delta \Longrightarrow\|f(x)-f(y)\| \leq K(\delta)\|x-y\| .
$$

(Such a $K(\delta)$ exists by Lemma 5.1.)

Obviously, $K(\delta)$ decreases as $\delta$ increases, and let $K=\lim _{\delta \rightarrow \infty} K(\delta)$. Then $K>0$. In fact, whenever $\|x-y\| \geq \delta>1,(*)$ and the definition of $K(\delta)$ imply that $\|f(x)-f(y)\| \leq K(\delta)\|x-y\| \leq K(\delta) L\|f(x)-f(y)\|$ so that $K \geq 1 / L$.

Fix $0<\epsilon<\frac{1}{2}$, to be specified at the end of the proof, and choose $\delta>1$ large enough so that $K(\delta) \leq(1+\epsilon) K$. Using the minimality of $K(2 \delta)$, fix $x, y \in X$ with $\|x-y\| \geq 2 \delta$ so that

$$
\|f(x)-f(y)\| \geq(1-\epsilon) K(2 \delta)\|x-y\| \geq(1-\epsilon) K\|x-y\| .
$$

Let $U=\{u \in X:\|u-x\|=\|u-y\|=\|x-y\| / 2\}$ be the set of metric midpoints between $x$ and $y$, and put

$$
\begin{aligned}
V_{\epsilon}=\{v \in Y:(1-4 \epsilon)\|f(x)-f(y)\| / 2 & \leq\|f(x)-v\|,\|f(y)-v\| \\
& \leq(1+4 \epsilon)\|f(x)-f(y)\| / 2\} .
\end{aligned}
$$


The set $V_{\epsilon}$ is the set of "almost" metric midpoints between $f(x)$ and $f(y)$.

We claim that $f(U) \subset V_{\epsilon}$. Indeed, fix $u \in U$, then

$$
\begin{aligned}
\|f(x)-f(u)\| & \leq K(\delta)\|x-u\| \\
& \leq \frac{1}{2}(1+\epsilon) K\|x-y\| \\
& \leq \frac{1}{2}(1+\epsilon)(1-\epsilon)^{-1}\|f(x)-f(y)\| \\
& \leq(1+4 \epsilon)\|f(x)-f(y)\| / 2
\end{aligned}
$$

(because $\epsilon<\frac{1}{2}$ ).

On the other hand, if we assume for contradiction that

$$
\|f(x)-f(u)\| \leq(1-4 \epsilon)\|f(x)-f(y)\| / 2
$$

we obtain

$$
\begin{aligned}
(1-\epsilon) K\|x-y\| & \leq\|f(x)-f(y)\| \\
& \leq\|f(x)-f(u)\|+\|f(u)-f(y)\| \\
& \leq(1-4 \epsilon)\|f(x)-f(y)\| / 2+\|f(u)-f(y)\| \\
& \leq K(\delta)\left((1-4 \epsilon) / 2+\frac{1}{2}\right)\|x-y\| \\
& \leq(1+\epsilon) K(1-2 \epsilon)\|x-y\| .
\end{aligned}
$$

So $(1-\epsilon) \leq(1+\epsilon)(1-2 \epsilon)$ which is impossible. Similarly

$$
(1-4 \epsilon)\|f(x)-f(y)\| / 2 \leq\|f(y)-f(u)\| \leq(1+4 \epsilon)\|f(x)-f(y)\| / 2 .
$$

We now specialize to $X=L_{1}$ and $Y=\ell_{1}$.

The set $U$ is a "large" subset of $L_{1}$ : It contains an infinite sequence $\left(x_{j}\right)$ so that $\left\|x_{j}-x_{k}\right\|=\|x-y\|$ for all $j \neq k$. Indeed, by translation and change of measure we can assume $x \equiv 0$ and $y \equiv a$ is constant, and then take $x_{j}=a\left(1+r_{j}\right) / 2$ where $\left(r_{j}\right)$ are the Rademacher functions.

On the other hand, $V_{\epsilon}$ is a "small" subset of $\ell_{1}$. A simple computation shows

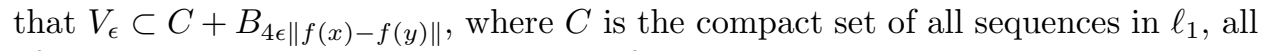
of whose coordinates lie between those of $x$ and $y$.

By the compactness of $C$, there must be $j \neq k$ so that $\left\|f\left(x_{j}\right)-f\left(x_{k}\right)\right\| \leq$ $10 \epsilon\|f(x)-f(y)\|$.

As $\left\|x_{j}-x_{k}\right\|=\|x-y\| \geq 2 \delta>1$, we obtain, using the definition of $L$, that

$$
\begin{aligned}
\|x-y\| & =\left\|x_{j}-x_{k}\right\| \leq L\left\|f\left(x_{j}\right)-f\left(x_{k}\right)\right\| \\
& \leq 10 \epsilon L\|f(x)-f(y)\| \leq 10 \epsilon L(1+\epsilon) K\|x-y\| .
\end{aligned}
$$

So we must have $10 \epsilon L(1+\epsilon) K \geq 1$, which does not hold if $\epsilon$ is small enough.

\section{§6. NON-ISOMORPhic, UNIFORMLY-HOMEOMORPHIC BANACH SPACES.}

In this section we present the recent examples of Ribe [Ri3] and Aharoni-Lindenstrauss [AL2] of uniformly homeomorphic Banach spaces which are not isomorphic. 
Theorem 6.1. Let $1 \leq p, q, p_{n}<\infty$ be such that $p_{n} \rightarrow p$. Then $\left(\sum \oplus \ell_{p_{n}}\right)_{q}$ is uniformly homeomorphic to $\ell_{p} \oplus\left(\sum \oplus \ell_{p_{n}}\right)_{q}$.

Note that when $p \neq q, p_{n}, \ell_{p}$ hence also $\ell_{p} \oplus\left(\sum \oplus \ell_{p_{n}}\right)_{q}$ does not even Lipschitz embed into $\left(\sum \oplus \ell_{p_{n}}\right)_{q}$.

As will be evident from the proof the method is quite flexible and one can prove similar results for other families of spaces, e.g., $\left(\sum \oplus L_{p_{n}}\right)_{q}$ is uniformly homeomorphic to $L_{p} \oplus\left(\sum \oplus L_{p_{n}}\right)_{q}$.

The Theorem was proved for $p=1$ by M. Ribe [Ri3]. I. Aharoni and J. Lindenstrauss [AL2] improved Ribe's techniques so as to yield the Theorem for general $1 \leq p<\infty$, so, in particular, by taking $q, p>1$ we obtain uniformly convex examples. Also, by taking $p=1$ and $q$ and $p_{n}$ all strictly greater than one, we obtain a reflexive space uniformly homeomorphic to a non-reflexive one.

The proof below is a technical simplification of the one in [AL2], obtained by carefully making all maps homogeneous. This avoids the need to deal simultaneously with the original norm and with other expressions, uniformly equivalent to it.

We start with some notation. In what follows $p$ and $q$ are fixed as in the Theorem. For fixed $1 \leq r, s<\infty$ the Mazur map (see [Maz]) $M_{r, s}: \ell_{r} \rightarrow \ell_{s}$ is defined by

$$
\left(M_{r, s}(x)\right)_{n}=\|x\|_{r}^{1-(r / s)} x_{n}^{r / s} \operatorname{sign} x_{n} .
$$

(Note that we added the normalizing factor $\|x\|_{r}^{1-(r / s)}$ so as to have a homogeneous map .) We shall use the following properties of $M_{r, s}$ :

$$
\left\|M_{r, s}(x)\right\|_{s}=\|x\|_{r} .
$$

For each $r, s, t$

$$
M_{r, t}=M_{s, t} \circ M_{r, s}
$$

and in particular

$$
M_{r, s}^{-1}=M_{s, r} .
$$

For each $r, s$ and each $K, M_{r, s}$ is a uniform homeomorphism of the $K$-ball in $\ell_{r}$ onto the $K$-ball in $\ell_{s}$. Moreover, the family $\left\{M_{r, s}: 1 \leq r, s<\infty\right\}$ where each $M_{r, s}$ is restricted to the ball of radius $\exp \left(|r-s|^{-1}\right)$ in $\ell_{r}$ is equi-uniformly-continuous. (For a proof of this last fact, see Lemma 1 in [Ri3].)

For each $r$, identify $\ell_{r}$ with $\ell_{r} \oplus_{r} \ell_{r}$, and define the map $I_{r}: \ell_{p} \oplus_{q} \ell_{r} \rightarrow \ell_{r}=$ $\ell_{r} \oplus_{r} \ell_{r}$ by

$$
I_{r}(x, y)=\frac{\|(x, y)\|}{\left\|\left(M_{p, r}(x), y\right)\right\|_{r}}\left(M_{p, r}(x), y\right) .
$$

Note that even when $r=p, I_{p}$ is not the identity but the renormalization of the norm in $\ell_{p} \oplus_{q} \ell_{p}$ to that of $\ell_{p} \oplus_{p} \ell_{p}$. Note also that in this case $I_{p}$ is a Lipschitz homeomorphism.

We shall need the following properties of $I_{r}$ :

$$
\left\|I_{r}(a)\right\|=\|a\| \text {. }
$$

For each fixed $r$ and $K, I_{r}$ is a uniform homeomorphism of the $K$-ball in $\ell_{p} \oplus_{q} \ell_{r}$ onto the $K$-ball of $\ell_{r}$. 
Moreover, the family $\left\{I_{r}: 1 \leq r<\infty\right\}$, where each $I_{r}$ is restricted to the ball of radius $\exp \left(|p-r|^{-1}\right)$ in $\ell_{p} \oplus_{q} \ell_{r}$ is equi-uniformly continuous.

To prove the Theorem one can obviously pass to a subsequence of the $p_{n}$ 's. We shall thus assume that $\exp \left(\left|p_{n}-p\right|^{-1}\right)>2^{n+1}$ for each $n=0,1,2, \ldots$.

The Theorem follows immediately from the following:

Proposition 6.2. There is a family of uniform homeomorphisms $\left\{F_{t}: 0 \leq t<\right.$ $\infty\}$ so that

(i) For $n=1,2, \ldots$ and $2^{n-1} \leq t \leq 2^{n}(0 \leq t \leq 1$ for $n=0)$, $F_{t}$ maps the ball of radius $t$ of $\ell_{p} \oplus_{q} \ell_{p_{n}} \oplus_{q} \ell_{p_{n+1}}$ onto the ball of radius $t$ of $\ell_{p_{n}} \oplus_{q} \ell_{p_{n+1}}$.

(ii) $\left\|F_{t}(a)\right\|=\|a\|$

(iii) $\left.F_{2^{n-1}}(x, y, z)=\left(I_{p_{n}}(x, y), z\right)\right)$ for $n=1,2, \ldots$

(iv) $F_{2^{n}}(x, y, z)=\left(y, I_{p_{n+1}}(x, z)\right)$ for $n=0,1, \ldots$

(v) The family $\left\{F_{t}(a), F_{t}^{-1}(a)\right\}$ is equi-uniformly continuous in both a and $t$, i.e., there is a function $\omega(\epsilon) \downarrow 0$ so that

$$
\left\|F_{t}(a)-F_{s}(b)\right\|,\left\|F_{t}^{-1}(a)-F_{s}^{-1}(b)\right\| \leq \omega(\|a-b\|+|t-s|)
$$

whenever there is an $n$ s.t. $2^{n-1} \leq t, s \leq 2^{n}$ and $a, b$ are such that all expressions make sense.

(Note that for $t=2^{k}, \quad F_{t}$ is defined twice - and in different ways. But this should cause no confusion, and in the application the two definitions will give rise to the same mapping.)

To deduce the Theorem from the Proposition define the homeomorphism $f$ : $\ell_{p} \oplus_{q}\left(\sum \oplus \ell_{p_{n}}\right)_{q} \rightarrow\left(\sum \oplus \ell_{p_{n}}\right)_{q}$ as follows:

If $a=\left(x, y_{0}, y_{1}, \ldots\right) \in \ell_{p} \oplus_{q}\left(\sum \oplus \ell_{p_{n}}\right)_{q}$ is such that $2^{n-1} \leq\|a\| \leq 2^{n}(0 \leq\|a\| \leq$ 1 for $n=0)$, define

$$
f(a)=\left(y_{0}, y_{1}, \ldots, y_{n-1}, F_{\|a\|}\left(x, y_{n}, y_{n+1}\right), y_{n+2}, \ldots\right)
$$

(with $F_{\|a\|}\left(x, y_{n}, y_{n+1}\right)$ occupying the $n^{\text {th }}$ and $(n+1)^{s t}$ coordinates).

Given $b=\left(z_{0}, z_{1}, \ldots\right) \in\left(\sum \oplus \ell_{p_{n}}\right)_{q}$, fixing $n$ so that $2^{n-1} \leq\|b\| \leq 2^{n}(n=$ 0 if $\|b\| \leq 1)$ determines what are the coordinates to be changed to obtain a $\epsilon \ell_{p} \oplus_{q}\left(\sum \oplus \ell_{p_{n}}\right)_{q}$ s.t. $f(a)=b$. Indeed, $a=\left(x, y_{0}, \ldots\right)$ is given by $y_{j}=z_{j}$ if $j \neq n, n+1$, and $\left(x, y_{n}, y_{n+1}\right)=F_{\|b\|}^{-1}\left(z_{n}, z_{n+1}\right)$. As all the maps are equiuniformly-continuous, and for $\|a\|=2^{m}$ the two different formulas obtained by writing $2^{m-1} \leq\|a\| \leq 2^{m}$ or $2^{m} \leq\|a\| \leq 2^{m+1}$ agree, $f$ is indeed a uniform homeomorphism.

Proof of Proposition. The proof has several steps. The first is a formal identification, reducing to a construction in spaces isomorphic to $\ell_{p}$. The second is a simplifying change of variable. In the third we construct homeomorphisms that do not preserve the norm, and in the last step we make the necessary normalization.

STEP I. The maps

$$
g_{n}: \ell_{p} \oplus_{q} \ell_{p_{n}} \oplus_{q} \ell_{p_{n+1}} \longrightarrow \ell_{p} \oplus_{q} \ell_{p} \oplus_{q} \ell_{p}
$$

and

$$
h_{n}: \ell_{p_{n}} \oplus_{q} \ell_{p_{n+1}} \longrightarrow \ell_{p} \oplus_{q} \ell_{p}
$$


given by

$$
\begin{aligned}
g_{n}(x, y, z) & =\left(x, M_{p_{n}, p}(y), M_{p_{n+1}, p}(z)\right) \\
h_{n}(x, y) & =\left(M_{p_{n}, p}(x), M_{p_{n+1}, p}(y)\right)
\end{aligned}
$$

preserve the norm and are equi-continuous uniform homeomorphisms when restricted to balls of radius $2^{n+1}$ in the respective spaces. As $F_{t}, 2^{n-1} \leq t \leq 2^{n}$ act on these balls only we shall take $F_{t}=h_{n}^{-1} \circ H_{t} \circ g_{n}$ where the maps

$$
H_{t}: \ell_{p} \oplus_{q} \ell_{p} \oplus_{q} \ell_{p} \longrightarrow \ell_{p} \oplus_{q} \ell_{p}
$$

will be constructed to satisfy (i-v) with $p$ replacing both $p_{n}$ and $p_{n+1}$.

STEP II. Fixing $n \geq 1$, (only notational changes are needed for $n=0$ ), we make a change of variable in $t$, so that it will vary in the interval $[0,1]$ rather than $2^{n-1} \leq t \leq 2^{n}$. Thus we shall construct homeomorphism

$$
G_{t}: \ell_{p} \oplus_{q} \ell_{p} \oplus_{q} \ell_{p} \longrightarrow \ell_{p} \oplus_{q} \ell_{p} \quad \text { for } \quad 0 \leq t \leq 1
$$

satisfying

(a) $\left\|G_{t}(a)\right\|=\|a\|$, and $G_{t}$ is homogenous

(b) $G_{0}(x, y, z)=\left(I_{p}(x, y), z\right)$

(c) $G_{1}(x, y, z)=\left(y, I_{p}(x, z)\right)$

(d) There is an absolute constant $K$ so that

$$
\left\|G_{t}(a)-G_{s}(b)\right\|,\left\|G_{t}^{-1}(a)-G_{s}^{-1}(a)\right\| \leq K(|t-s|+\|a-b\|)
$$

provided $\|a\|,\|b\| \leq 1$.

Once this is done define for $2^{n-1} \leq t \leq 2^{n} \quad H_{t}(a)=G_{t 2^{1-n}-1}(a)$, and then using the homogeneity of the $G$ 's, we have, whenever $\|a\|,\|b\| \leq 2^{n}$ that

$$
\begin{aligned}
\left\|H_{t}(a)-H_{s}(b)\right\| & =2^{n}\left\|G_{t 2^{1-n}-1}\left(2^{-n} a\right)-G_{s 2^{1-n}-1}\left(2^{-n} b\right)\right\| \\
& \leq 2^{n} K\left(|t-s| 2^{1-n}+2^{-n}\|a-b\|\right) \\
& \leq 2 K(|t-s|+\|a-b\|)
\end{aligned}
$$

i.e., $H_{t}(a)$ is equi Lipschitzian in both $a$ and $t$, and similarly for $H_{t}^{-1}(a)$.

STEP III. Consider the operators $S_{0}, S_{1}: \ell_{p} \oplus_{q} \ell_{p} \oplus_{q} \ell_{p} \longrightarrow \ell_{p} \oplus_{q} \ell_{p}$ given by

$$
\begin{aligned}
& S_{0}(x, y, z)=(\langle x, y\rangle, z) \\
& S_{1}(x, y, z)=(y,\langle x, z\rangle)
\end{aligned}
$$

where, in the definition of $S_{0}$, we identify the first copy of $\ell_{p}$ in $\ell_{p} \oplus_{q} \ell_{p}$ with $\ell_{p} \oplus_{q} \ell_{p}$ and write $\langle x, y\rangle$ for the general point in $\ell_{p}$ represented this way. Similarly, in the definition of $S_{1}$, it is the second copy of $\ell_{p}$ in $\ell_{p} \oplus_{q} \ell_{p}$ which is represented in this way.

The two spaces $\ell_{p} \oplus_{q} \ell_{p} \oplus_{q} \ell_{p}$ and $\ell_{p} \oplus_{q} \ell_{p}$ are both isomorphic to $\ell_{p}$, and the general linear group of $\ell_{p}$ is contractible. As both $S_{0}$ and $S_{1}$ are isomorphisms, there is a continuous map $t \rightarrow S_{t}$, where for each $0 \leq t \leq 1, S_{t}$ is an invertible 
operator from $\ell_{p} \oplus_{q} \ell_{p} \oplus_{q} \ell_{p}$ onto $\ell_{p} \oplus_{q} \ell_{p}$, and a constant $K$, so that $S_{0}$ and $S_{1}$ are the given operators and

$$
\left\|S_{t}\right\|,\left\|S_{t}^{-1}\right\| \leq K
$$

for all $0 \leq t \leq 1$.

Moreover, using the continuity of $t \rightarrow S_{t}$ and the compactness of [0,1], we can approximate, and then replace the $S_{t}$ 's with a map $t \rightarrow S_{t}$ which is Lipschitz in $t$ (in fact, even piecewise linear). So we can also assume

$$
\left\|S_{t}-S_{s}\right\|,\left\|S_{t}^{-1}-S_{s}^{-1}\right\| \leq K|t-s| .
$$

STEP IV. The operators $S_{t}$ almost do the job as the required $G_{t}$. They are, however, only isomorphisms and we need to preserve the norm, i.e., to renormalize them. But note that we need special renormalizations for $t=0$ and $t=1$. Indeed, $S_{0}(x, y, z)=(\langle x, y\rangle, z)$ and we need to have $G_{0}(x, y, z)=\left(I_{p}(x, y), z\right)$, i.e., for $t=0$, we need to renormalize the first coordinate of $\ell_{p} \oplus_{q} \ell_{p}$. Similarly, for $t=1$, we need to renormalize the last coordinate only. Thus we shall need to do the renormalizations differently for different values of $t$, and it will be more convenient to work with the interval $-1 \leq t \leq 2$ rather than $0 \leq t \leq 1$, (i.e., we define $G_{t}$ for $-1 \leq t \leq 2$, and require in (b) that $G_{-1}(x, y, z)=\left(I_{p}(x, y), z\right)$ and in (c) that $G_{2}(x, y, z)=\left(y, I_{p}(x, z)\right)$.)

For $0 \leq t \leq 1$, we define $G_{t}(a)=\|a\| \frac{S_{t}(a)}{\left\|S_{t}(a)\right\|}$. Then $G_{t}^{-1}(b)=\|b\| \frac{S_{t}^{-1}(b)}{\left\|S_{t}^{-1}(b)\right\|}$ and (a) and (d) are satisfied.

Writing $a=(x, y, z)$ we define for $-1 \leq t \leq 0$

$$
\begin{aligned}
G_{t}(a) & =\|a\| \frac{-t\left(I_{p}(x, y), z\right)+(1+t) S_{0}(a)}{\left\|-t\left(I_{p}(x, y), z\right)+(1+t) S_{0}(a)\right\|} \\
& =\|a\| \frac{\left(\psi_{t}(x, y)\langle x, y\rangle, z\right)}{\left\|\left(\psi_{t}(x, y)\langle x, y\rangle, z\right)\right\|}
\end{aligned}
$$

where

$$
\psi_{t}(x, y)=-t \frac{\left(\|x\|^{q}+\|y\|^{q}\right)^{1 / q}}{\left(\|x\|^{p}+\|y\|^{p}\right)^{1 / p}}+(1+t) .
$$

Then $G_{-1}(x, y, z)=\left(I_{p}(x, y), z\right)$ (because $\left.\left\|\left(I_{p}(x, y), z\right)\right\|=\|a\|\right)$, and this formula for $G_{0}(a)$ agrees with the previous one, i.e.,

$$
G_{0}(a)=\|a\| \frac{S_{0}(a)}{\left\|S_{0}(a)\right\|} .
$$

If $b=(u, v) \in \ell_{p} \oplus_{q} \ell_{p}$ then

$$
G_{t}^{-1}(b)=\|b\| \frac{\left(\frac{1}{\psi_{t}(u)} u, v\right)}{\left\|\left(\frac{1}{\psi_{t}(u)} u, v\right)\right\|}
$$

(where $\psi_{t}(u)$ is defined by the above formula upon writing $u=\langle x, y\rangle$ ).

It is a straightforward computation to check that $G_{t}$ satisfy also (d) for $-1 \leq$ $t \leq 0$.

A similar formula works for $1 \leq t \leq 2$ :

$$
G_{t}(a)=\|a\| \frac{(2-t) S_{1}(a)+(t-1)\left(y, I_{p}(x, z)\right)}{\left\|(2-t) S_{1}(a)+(t-1)\left(y, I_{p}(x, z)\right)\right\|} .
$$




\section{REFERENCES}

[Ah] I. Aharoni, Every separable Banach space is Lipschitz equivalent to a subset of $c_{0}$, Israel J. Math. 19 (1974), 284-291.

[AL1] I. Aharoni and J. Lindenstrauss, Uniform equivalence between Banach spaces, Bull. Amer. Math. Soc. 84 (1978), 281-283..

[AL2] - An extension of a result of Ribe, preprint.

$[\mathrm{AMM}]$ I. Aharoni, B. Maurey and B.S. Mitjagin, Uniform embedding of metric spaces and of Banach spaces into Hilbert space (to appear).

[Ar] N. Aronszajn, Differentiability of Lipschitzian mappings between Banach spaces, Studia Math. 58 (1976), 147-190.

[As] P. Assouad, Remarques sur un article de Israel Aharoni sur les prolongements Lipschitziens dans $c_{0}$, Israel J. Math. 31 (1978 97-100).

[BP] C. Bessage and A. Pełczyǹski, Selected topics in infinite dimensional topology, PWN, Warszawa, 1975.

[Bi] P. Billengsly, Convergence of Probability Measures, John Wiley \& Sons, 1968.

[Bo] J. Bourgain, On Lipschitz embedding of finite metric spaces in Hilbert spaces (to appear).

[BMW] J. Bourgain, V.D. Milman and H. Wolfson, On type of metric spaces (to appear).

[C] J.P.R. Christensen, Measure theoretic zero sets in infinite dimensional spaces and applications to differentiability of Lipschitz mappings, II, Coll. Anal. Funct., Bordaux (1973), 29-39.

[E1] P. Enflo, On the non-existance of uniform homeomorphisms between $L_{p}$-spaces, Ark. Mat. 8 (1969), 103-105.

[E2] — On a problem of Smirnov, Ark. Mat. 8 (1969), 107-109.

[E3] Uniform structures and square roots in topological groups Part II, Israel J. Math. 8 (1970), 253-272.

[E4] Uniform homeomorphisms between Banach spaces, Seminaire Maurey-Schwartz , 1975-1976, exposé 18.

[G] I.M. Gelfand, Abstrakte Funktionen und lineare operatoren, Mat. Sb. (N.S.) 4 (1938), no. $46,235-286$.

[HM] S. Heinrich and P. Mankiewicz, Applications of ultrapowers to the uniform and Lipschitz classification of Banach spaces, Studia Math. 73 (1982), 225-251.

[JL] W.B. Johnson and J. Lindenstrauss, Extensions of Lipschitz mappings into a Hilbert space, Conference in Modern Analysis and Probability, Yale 1982, Contemporary Math. 26.

[L] J. Lindenstrauss, On non-linear projections in Banach spaces, Mich. J. Math. 11 (1964), 268-287.

[Man] P. Mankiewicz, On the differentiability of Lipschitz mappings in Feréchet spaces, Studia Math. 45 (1973), 15-29.

[Maz] S. Mazur, Une remarque sur l'homéomorphismie des champs fonctionnels, Studia Math. 1 (1930), 83-85.

[P] R.R. Phelps, Gaussian null sets and differentiability of Lipschitz maps on Banach spaces, Pacific J. Math. 77 (1978), 523-531.

[Ra] H. Rademacher, Über partielle und totale Differenzierbarkeit von funktionen mehrerer variablen und über die transformation der doppelintegrale, Math. Ann. 79 (1919), 340359.

[Ri1] M. Ribe, On uniformly homeomorphic normed spaces, Ark. Math. 14 (1976), 237-244.

[Ri2] _ On uniformly homeomorphic normed spaces, II, Ark. Math. 16 (1978), 1-9.

[Ri3] Existance of separable uniformly homeomorphic non isomorphic Banach spaces, Israel J. Math. 48 (1984), 139-147.

[T] H. Torunczyk, Characterizing Hilbert space topology, Fund. Math. 111 (1981), 247-262. 